\title{
Aprendizaje de la Teoría de la Relatividad Restringida de Einstein. Estado de la Cuestión
}

\author{
Xabier Prado \\ Departamento de Didácticas Aplicadas, Facultade de Ciencias da Educación. Universidade de Santiago de \\ Compostela.15782-Santiago de Compostela.España.xabier.prado@gmail.com \\ ORCID: https:// orcid.org/0000-0001-9535-7499
}

José Manuel Domínguez-Castiñeiras

Departamento de Didácticas Aplicadas, Facultade de Ciencias da Educación. Universidade de Santiago de Compostela.15782-Santiago de Compostela.España.josemanuel.dominguez@usc.es

ORCID: bttps:/ / orcid.org/0000-0002-5159-8968

Iván Area

Departamento de Matemática Aplicada II, Escola de Enxeñaría de Telecomunicación. Universidade de

Vigo.32004-Ourense.España.area@uvigo.es

ORCID: https:// orcid.org/0000-0003-0872-5017

Ángel Paredes

Departamento de Física Aplicada, Escola de Enxeñaría Aeronútica e do Espazo. Universidade de Vigo.

32004-Ourense.España.angel.paredes@uvigo.es

ORCID: https:/ / orcid.org/0000-0003-3207-1586

\section{Jorge Mira}

Departamento de Física Aplicada, Facultade de Física. Universidade de Santiago de Compostela. 15782-

Santiago de Compostela.jorge.mira@usc.es

ORCID: bttp:/ / orcid.org/0000-0002-6024-6294

[Recibido: 30 Julio 2019. Revisado: 27 Octubre 2019. Aceptado: 16 Diciembre 2019]

Resumen: En este artículo se analizan las dificultades de aprendizaje de la Teoría de la Relatividad Restringida de Einstein. La información se ha obtenido y seleccionado de las bases de datos Web of Science, Scopus, Educational Resources Information Center y Google Scholar, teniendo en cuenta los índices de valoración Journal Citation Reports (JCR), Scimago Journal Rank (SJR) e Índice Compuesto de Difusión Secundaria (ICDS).

Para el análisis y las conclusiones se establecieron las dificultades de aprendizaje del alumnado relacionadas con la Relatividad Restringida de Einstein, respecto a los siguientes conceptos: Espacio y tiempo. Causalidad y simultaneidad. Sistemas de referencia. Energía. Masa. Luz.

El presente estudio ha constituido el fundamento del diseño de una propuesta de enseñanza basada en la formulación geométrica de Minkowski, dirigida a estudiantes de bachillerato.

Palabras clave: Relatividad restringida de Einstein. Geometría de Minkowski. Dificultades de aprendizaje. Revisión bibliográfica.

\section{Learning Einstein's Theory of Special Relativity: State Of the Art}

Abstract: In this article we analyze the learning difficulties of Einstein's Theory of Restricted Relativity. The information has been obtained and selected from the Web of Science, Scopus, Educational Resources Information Center and Google Scholar databases, taking into account the Journal Citation Reports (JCR), Scimago Journal Rank (SJR) and Composite Index of Secondary Dissemination (ICDS).

For the analysis and the conclusions the learning difficulties of the students related to the Restricted Relativity of Einstein were established, with respect to the following concepts: Space and time. Causality and simultaneity. Reference frames. Energy. Mass. Light. 
This study has been the basis for the design of a teaching proposal based on the geometric formulation of Minkowski, aimed at high school students.

Keywords: Einstein's spcaeli relativity. Geometry of Minkowski. Learning difficulties. Bibliographic review.

Para citar este artículo: Prado X., Dominguez-Castiñeiras J.M., Area I., Paredes A., y Mira J. (2020) Aprendizaje de la Teoría de la Relatividad Restringida de Einstein. Estado de la Cuestión. Revista Eureka sobre Enseñanza y Divulgación de las Ciencias 17(1), 1103. doi: 10.25267/Rev_Eureka_ensen_divulg_cienc.2020.v17.i1.1103

\section{Introducción}

La teoría de la Relatividad Restringida (en lo sucesivo, TRR), publicada en 1905, versa sobre la comparación de observaciones entre dos sistemas de referencia diferentes circunscribiendo el análisis de la teoría más general desarrollada por Einstein en 1915 a aquellos casos en los que la curvatura del espaciotiempo producida por la acción de la gravedad sea irrelevante. Se la conoce también como relatividad especial, aunque en este trabajo se opta por el adjetivo restringida (Consello da Cultura Galega 2017), más fiel a la denominación alemana original: Spezielle Relativitätstheorie. Señalaremos que dicha teoría ha sido ampliamente analizada en la didáctica de las ciencias.

El objetivo del presente trabajo es obtener una visión global sobre la problemática del aprendizaje de la TRR, a la luz de la investigación realizada hasta el momento.

Con la información obtenida de este estudio se ha fundamentado el diseño de una propuesta didáctica (Prado Orbán y Domínguez-Castiñeiras 2010) basada en la formulación geométrica de Minkowski (Corry 1997; Walter 2008; Minkowski 2012; Naber 2012), para la enseñanza de la TRR en el bachillerato (Prado Orbán 2012). Esta revisión bibliográfica podría permitir el acceso al conocimiento elaborado con anterioridad y ahorrar tiempo y esfuerzo para seguir investigando e innovando. Este trabajo puede clasificarse en las siguientes líneas de investigación: Desarrollo del pensamiento científico, y Diseño, implementación y evaluación de propuestas de enseñanza que utilizan la geometría de Minkowski.

\section{Método}

Para la selección de la bibliografía analizada se han utilizado las bases de datos y establecido los criterios que se indican a continuación.

\section{Recuperación de la información. Fuentes documentales}

Para la búsqueda de la literatura nos hemos servido de las bases de datos Web of Science, Scopus, Educational Resources Information Center y Google Scholar. Están especializadas en ciencias humanas y sociales y contienen amplia información sobre revistas y recursos relacionados con la educación.

\section{Análisis de la fiabilidad y validez de la información. Criterios de selección}

Los criterios de selección vienen determinados por:

El objetivo de la revisión: obtener una visión global sobre la problemática del aprendizaje de la TRR de Einstein.

La fiabilidad y validez de los artículos: contrastados por los índices de valoración Journal Citation Reports (JCR), Scimago Journal Rank (SJR) e Índice Compuesto de Difusión Secundaria (ICDS). 


\section{Resultados y Discusión}

El principio de relatividad, establecido en su versión clásica por Galileo, plantea dilemas epistemológicos, como la atribución de un carácter absoluto al reposo (Hewson 1982), que deben ser objeto de un tratamiento específico antes de abordar la teoría de la relatividad de Einstein.

Los problemas de aprendizaje de la TRR en los niveles más básicos obedecen a varios factores, entre los que cabe destacar la dificultad específica que tiene apropiarse de determinados conceptos de esta teoría; la metodología habitualmente empleada para desarrollar el tema y, en los niveles más básicos, las carencias del propio profesorado.

Por lo que se refiere a la teoría en sí, la complejidad de determinados conceptos relacionados con la TRR radica, sobre todo, en la necesidad de realizar una nueva interpretación de fenómenos que intuitivamente se consideran "simples". Cuando se aborda la enseñanza de la teoría, cabe esperar la existencia o aparición de ideas alternativas en el alumnado. Aslanides y Savage (2013) probaron un cuestionario sobre el concepto de relatividad para evaluar la comprensión de los estudiantes de los conceptos relativistas, y Otero, Arlego y Prodanoff (2016) desarrollaron una estructura conceptual de referencia para relacionar de forma visual los distintos conceptos subyacentes a la TRR. En cuanto a la metodología empleada para desarrollar este tema, los análisis realizados de los libros de texto y otros materiales didácticos (Pérez y Solbes 2003; Alonso y Soler 2002) muestran que es muy habitual que, tras señalar la incompatibilidad de las leyes de la mecánica de Newton con algunos hechos que impulsaron el desarrollo de la TRR (por ejemplo, la existencia de una velocidad límite), se emprenda un proceso de modificación de algunas de las leyes newtonianas para construir los nuevos conceptos. A ojos de los alumnos, esta práctica reiterada puede parecer un intento vano de mantener algo de los conceptos que se han de superar. Como veremos en los apartados que siguen, es frecuente que los estudiantes solapen conceptos de ambas teorías y no perciban la TRR como una teoría autónoma. Por ello, defendemos (Alonso y Soler 2006 a) que una presentación correcta de la TRR debería deducir directamente todas sus leyes y conceptos de los postulados de Einstein y luego mostrar, eso sí, el grado de error o margen de licitud tolerable al utilizar la mecánica newtoniana (el límite newtoniano, cuando $v / c$ ).

En cuanto a la labor docente, se han puesto de manifiesto errores crasos fuertemente arraigados entre el profesorado de Bachillerato (Alemañ 1997), la mayoría de los cuales obedecen a la tendencia al deslizamiento hacia una explicación de las ideas relativistas basada en nociones newtonianas — con la consiguiente confusión de conceptos- y a la inadecuada interpretación de fenómenos cuya significación varía radicalmente de una teoría a otra.

Numerosos autores han examinado esta problemática desde la segunda mitad del siglo XX. Se ha sistematizado en las siguientes categorías: Espacio y tiempo. Causalidad y simultaneidad. Sistemas de referencia. Energía. Masa. Lu\%.

\section{Espacio y tiempo}

Una teoría que cuestiona ideas tan arraigadas en la propia percepción humana como estas tenía que encontrar serias resistencias para su aceptación, tal como ponen de manifiesto Hewson y Thorley (1989): la idea de tiempo absoluto permanece incuestionable, como aquello que fluye por igual para todos y en todas partes, en una formulación muy similar a la establecida por el propio Newton en sus axiomas. Ello señala el problema didáctico causado por dichas ideas, pues precisamente una de las consecuencias de la teoría de la relatividad es la desaparición de la noción de tiempo absoluto. 
A este respecto, Villani y Pacca (1987) constataron que los estudiantes tienen problemas para aceptar que las medidas de longitud y tiempo pueden depender del sistema de referencia y, en general, atribuyen un carácter absoluto a las medidas realizadas en un sistema de referencia en reposo con respecto al observador.

Pérez (2003) indica que en este caso la concepción newtoniana (principio de Relatividad de Galileo) se toma como punto de partida y la visión relativista que surge de la TRR (principio de relatividad de Einstein), como el de llegada. Sin embargo, y dado que todos los esfuerzos en los niveles anteriores se han dirigido a consolidar la concepción newtoniana antes mencionada frente a los esquemas espontáneos del alumnado - conceptos de espacio y tiempo intuitivos y muy poderosos, enraizados en la mente y en la psicología humana hasta el punto de que el propio Kant razonó acerca de la verdad intrínseca de los mismos-, resulta que los estudiantes se enfrentan a la superación de este marco newtoniano que tanto esfuerzo les costó adquirir y consolidar, y sin contar con la posibilidad de experiencias cotidianas que les permitan aceptar la posibilidad de la nueva teoría o corroborar su eficiencia.

Toledo, Arriasseco y Santos (1997) muestran que no es posible sustituir directamente los conceptos de la mecánica newtoniana por los nuevos conceptos relativistas después de una instrucción formal, sino que se unen a ellos en una interacción compleja y sin anclajes firmes que permitan resolver situaciones diferentes de las tratadas en la instrucción. Gil y Solbes (1993) señalan que la persistencia y la estabilidad de las concepciones relativistas son muy bajas.

En resumen, para asumir la TRR hay que sustituir los conceptos de espacio y tiempo absolutos de la mecánica de Newton por los nuevos conceptos relativistas donde espacio y tiempo son interdependientes, mientras que el módulo del intervalo espaciotiempo es absoluto. Se trata de un requisito irrenunciable para aprender el propio concepto de relatividad, que puede ser causa de confusión si no se explica adecuadamente. Es interesante, a este respecto, que Minkowski se sintiese incómodo con la elección del término relatividad por parte de Einstein para referirse a una teoría que, como él mismo había demostrado (Minkowski 2012), trata esencialmente con las propiedades geométricas de un espaciotiempo absoluto.

Taylor (2001) discutió las ideas de Leibnitz y Newton con respecto al tiempo. Newton previó un tiempo en flujo permanente, incluso antes de la creación del universo. En agudo contraste, Leibnitz concibió el tiempo como una relación entre los eventos y que, por lo tanto, no tendría sentido en su ausencia o en ausencia del universo donde tienen lugar. El espacio, consecuentemente, se concibe a partir de las relaciones entre los objetos y, por lo tanto, dejaría de tener sentido si solamente existiese un objeto en todo el universo.

Doménech, Doménech, Cassasus y Bella (1985) realizan un análisis que compara el espaciotiempo de la TRR (interdependiente) con el espacio y el tiempo de la mecánica de Newton (absolutos). Presentan una relación de propiedades de las magnitudes citadas, como son la de pasividad, independencia, integridad, continuidad, homogeneidad e isotropía. En el caso de la mecánica newtoniana, añaden las hipótesis de universalidad temporal, la invariancia de las distancias espaciales y el carácter euclidiano del espacio, mientras que, para el caso de la TRR, esas hipótesis se sustituyen por las de relatividad de los intervalos espaciales y temporales dentro de un espaciotiempo pseudoeuclidiano, siguiendo el modelo geométrico propuesto por Minkowski.

\section{Causalidad y simultaneidad}

La TRR desafía el concepto de tiempo absoluto y, como consecuencia de ello, la noción de simultaneidad se altera y también deja de ser absoluta. En el marco de la TRR dos eventos que 
sean simultáneos en un sistema referencial inercial (SRI), no lo son en ningún otro: en ciertos SRI uno de ellos ocurre antes que el otro, mientras que en otros SRI se invierte el orden temporal entre esos mismos eventos. Este carácter relativo de la simultaneidad puede sugerir un cuestionamiento del concepto de causalidad, también conocido como relación causa/efecto. Es natural preguntarse si las relaciones causa/efecto pierden su carácter absoluto y si la causa y el efecto pueden intercambiarse al considerar diferentes sistemas de referencia. Por supuesto que esto no ocurre en la TRR y, por lo tanto, constituye una verificación de la coherencia de la teoría.

En el entramado espaciotemporal de la TRR, cabe considerar dos tipos de relación entre cualquier pareja de eventos. Una posibilidad es que dichos eventos se puedan ligar causalmente, es decir, que uno de ellos pueda influir en el otro o ser causa de él, para lo que se requiere que sea posible enviar una señal, por supuesto, con velocidad inferior o igual a la de la luz, desde uno (anterior) hasta el otro (posterior). Así, el primero (causa) es absolutamente anterior al segundo (efecto), es decir, la causa ocurre antes que el efecto en todos los SRI. La segunda posibilidad es que los eventos sean causalmente inconexos. En este caso existe un único SRI en el que ambos son simultáneos, mientras que su orden temporal puede alterarse dependiendo de otros SRI alternativos que se adopten.

Esta conexión/desconexión causal de los eventos en la TRR implica sutilezas conceptuales que eventualmente se convierten en dificultades para su aprendizaje. Scherr, Shaffer y Vokos (2001) investigaron la comprensión de los conceptos de tiempo y simultaneidad en la TRR por estudiantes universitarios de grados científicos y no científicos. Presentaron una relación de errores sobre los citados conceptos: la creencia de que dos eventos son simultáneos si un observador recibe señales de ambos al mismo tiempo, la convicción de que la simultaneidad es absoluta y la suposición de que cada observador constituye un sistema de referencia diferente. A continuación, Scherr, Shaffer y Vokos (2002) propusieron una serie de estrategias para evaluar y resolver las ideas alternativas. En Bachillerato, Alonso (2000) y Alonso y Soler (2006 b) desarrollaron una secuencia didáctica que utiliza los diagramas espaciotiempo de Minkowski para clarificar estos conceptos de simultaneidad y de causalidad.

\section{Sistemas de referencia}

Asumir la equivalencia de diversos sistemas de referencia requiere haber superado la tendencia espontánea de suponer sistemas de referencia privilegiados. Si este avance no se ha producido en el marco de la mecánica de Newton, la noción sobre sistemas de referencia privilegiados persiste en la TRR, e incluso influye en la percepción de lo que es real o no.

McDermott (1993), al comparar cómo enseña el profesorado y cómo aprenden los estudiantes en el marco de la mecánica newtoniana, observa la dificultad que tienen estos para relacionar las gráficas espaciotiempo con los movimientos que representan y los conceptos de energía y cantidad de movimiento, haciendo énfasis en la necesidad de desarrollar estas habilidades.

Hewson (1982) analizando las explicaciones de los fenómenos relativistas, llega a la conclusión de que los estudiantes asumen como real solo lo que está en reposo con respecto a sí mismos. Las medidas realizadas sobre algo que se mueve (por ejemplo, la vida media de una partícula, o una longitud) no se corresponden con las de un cuerpo en reposo: parece que la longitud y el tiempo son diferentes, pero se interioriza la existencia de una realidad absoluta asociada al reposo.

Cuando los estudiantes se enfrentan a la necesidad de explicar fenómenos relativistas a partir de diferentes sistemas de referencia, recurren a veces a analogías con conceptos que ya encontraron al estudiar una situación diferente (Hartatiek y Dwi 2017). Un ejemplo de este procedimiento es el uso de la paradoja de los gemelos para abordar de forma errónea 
problemas analógicos sobre las comparaciones de tiempo entre diferentes sistemas de referencia (Tanel 2014).

Para concluir este apartado sobre los sistemas de referencia, conviene añadir una llamada de atención acerca de la creencia errónea de que la TRR sólo puede tratarse en sistemas de referencia inerciales (SRI). Las leyes más elementales de la TRR (las que se ven en las etapas más básicas como el Bachillerato) se refieren, en efecto, al cambio de algunas magnitudes entre sistemas inerciales y también es cierto que Einstein consideró, antes del desarrollo de la relatividad general, casi exclusivamente sistemas de coordenadas relacionados por transformaciones de Lorentz (SRI). Ahora bien, varios autores (Logunov 1998) han mostrado, que la TRR puede tratar igualmente sistemas de referencia no inerciales, aunque ese tratamiento es mucho más complicado y lógicamente queda fuera de estas etapas educativas.

Hasta ahora se han descrito problemas de aprendizaje de la TRR que incluyen conceptos de cinemática relativista, apuntando que para contribuir a su superación conviene un tratamiento geométrico cuatridimensional, implementando expresamente dichos conceptos en el entramado espaciotemporal. Seguidamente repasaremos algunos de los problemas de aprendizaje que contienen conceptos de dinámica relativista que es posiblemente el capítulo peor (y más escasamente) tratado en la enseñanza de esta teoría en los niveles más básicos. De hecho, en bachillerato sus leyes o expresiones se presentan a menudo (en los libros de texto y en el aula) sin mediar justificación formal alguna y no se muestra ninguna ligazón entre ellos y los postulados de la TRR (Alonso y Soler 2002).

\section{Energía}

Cuando se enseña la mecánica de Newton, normalmente no se dedica tiempo ni atención a ver que, mientras en esta teoría magnitudes como la energía cinética, por ejemplo, son relativas (su valor depende del SRI), los incrementos de energía son absolutos, y que, por ello, se puede usar y se usa dicho principio de conservación de la energía como una ley fundamental o punto de partida para resolver problemas de dinámica newtoniana. No tratar estos conceptos hace más difícil apropiarse luego de los de la TRR.

En el aula, rara vez se contempla la relación entre los intercambios de energía y los sistemas de referencia, especialmente cuando se trata de energía cinética o trabajo (Galili y Kaplan 1997). Díaz, Herrera y Manjarrés (2009) señalan que el trabajo y la energía difieren desde el punto de vista de sistemas de referencia clásicos dispares.

Fort (2005) dio un paso más y presentó una aplicación del cambio de sistema de referencia para incorporar el tiempo en las relaciones energéticas, un enfoque que generalmente se ignora al resolver problemas de dinámica teniendo en cuenta la conservación de la energía. De esta forma, además de ampliar el rango de aplicabilidad del tratamiento energético de los problemas de dinámica, las ideas relativistas se corroboran, ganando credibilidad. Tefft y Tefft (2007) señalan la necesidad de discutir específicamente los efectos relativistas relacionados con la energía desde la perspectiva de la relatividad de Galileo (mecánica de Newton), como un paso previo a la enseñanza de la TRR.

Para discutir la diferencia entre los conceptos de energía cinética y momento, Riggs (2016) aboga por una presentación que minimice el formalismo matemático, tanto para la mecánica newtoniana como para la relatividad restringida. Por otro lado, Dib (2013) y Serafin y Glazek (2017) discuten la contribución de las fuerzas de enlace (asociadas a la energía potencial) a la masa-energía total de un sistema de partículas. 


\section{Masa}

El concepto de masa en la relatividad ha venido adoleciendo de graves dificultades, en buena medida propiciadas por la inercia de mantener una terminología que usaba una masa dependiente de la velocidad, en lugar de adoptar la terminología estándar actual, donde se considera la masa invariante, proporcional al módulo del cuadrivector impulso-energía.

Los estudiantes trabajan con nociones diversas en relación con la masa, dependiendo del contexto (Doménech, Casasús y Doménech 1993): cantidad de materia (asociada a la noción de átomos); principio de conservación (asociado a la química); masa dependiente de la velocidad (asociada a contextos de ciencia ficción); desaparición de masa en reacciones nucleares, etc. Una definición en términos de masa como cantidad de materia da lugar a dificultades para identificar la masa en un sistema inmaterial como un par de fotones que se alejan entre sí (Pérez y Solbes 2003). El concepto de masa relativista también fue criticado por Okun (1989) y es curioso que incluso dé lugar a confusiones en la literatura científica actual (Haug 2017; Tommasini, Paredes y Michinel 2019).

El concepto de masa relativista, o masa dependiente de la velocidad, supone una complicación adicional debido a su contraste con la noción aceptada de masa invariante o propia. El uso del concepto de masa relativista conduce a conceptos confusos como el de masa transversal y masa longitudinal, que difieren entre sí para una partícula dada en un sistema de referencia determinado (Pérez y Solbes 2006). Además, la masa relativista se centra en las partículas más que en las propiedades intrínsecas del espaciotiempo, como sería deseable (Whitaker 1976).

Warren (1976) destacó las nociones erróneas de desaparición y aparición de masa en reacciones nucleares, cuyo origen radica en la confusión entre la suma de los componentes de un sistema, que puede cambiar, y su masa invariante total que permanece como una constante.

Estas consideraciones fueron el centro de un debate (Doménech 1998) sobre la noción de masa relativista (o masa dependiente de la velocidad), concepto cada vez menos aceptado puesto que correspondería físicamente a la energía total incluyendo la masa invariante y la energía cinética. Alemañ (1997) explica que, para cambiar la velocidad de una partícula, no es necesario ejercer ninguna acción sobre ella, sino que simplemente bastará con cambiar el sistema de referencia (SR) desde el que se determina dicha velocidad. Una partícula tiene velocidades diferentes desde SR distintos, sin que a ella (ni a su masa, de ahí su característica de invarianza) le pase nada (sí es diferente, en cambio, en cada SR, su impulso y su energía).

Hecht (2009) sostiene que Einstein nunca estuvo a favor del concepto de masa relativista y prefirió el concepto de masa invariante, que equivale a la masa en reposo de una sola partícula y a la energía total en el sistema de referencia del centro de masas de un conjunto de partículas.

Hobson $(2005,2006)$ propone presentar la fórmula de Einstein considerando que la masa equivale a la energía del campo subyacente a cada partícula y, para los sistemas de partículas, a la energía cinética térmica general. Hecht (2011) analiza los problemas a los que tuvo que enfrentarse Einstein para establecer la ecuación, lo que permite anticipar los que podrían encontrar los estudiantes.

\section{Luz}

La idea de velocidad límite está estrechamente ligada a la teoría de la relatividad y se considera virtualmente consubstancial a ella. Sin embargo, de nuevo nos encontramos con el obstáculo de que los estudiantes razonan sobre problemas complejos con argumentos de carácter lógico formal, sin recurrir a diagramas espaciotemporales en los que las situaciones podrían encajar y ser resueltas adecuadamente. 
Analizar la validez y la universalidad del fenómeno relativista de la velocidad límite, junto con las dificultades para comprender los conceptos asociados, se considera esencial. En este sentido, se realizaron experimentos (Salart, Baas, Branciard, Gisin y Zbinden 2008) para verificar la existencia física de las velocidades superlumínicas. Se sabe que, por ejemplo, una sombra, un punto láser en la superficie de la Luna, o un fotón en regiones particulares del Universo en expansión (Drummond y Hathrell 1980) pueden moverse más rápidos que la luz sin que ello implique una violación física del principio de velocidad límite. Esto puede llevar a los estudiantes a creer que experimentos como los indicados prueban que la teoría de la relatividad es, en sí misma, relativa y que con el avance de la ciencia podría lograrse superar la limitación de velocidad.

Villani y Pacca (1987) señalaron que, en cuestiones relacionadas con la velocidad de la luz, prevalecen las ideas de movimiento absoluto y las nociones fluctuantes entre lo real y lo ficticio. Ello es cierto incluso entre estudiantes universitarios, y en el siguiente argumento surgen consistentemente: dos observadores parecen medir valores diferentes mientras que, en realidad, existe una sola cuantificación de espacio, tiempo y velocidad de la luz. Debido al alto valor de la velocidad de la luz en comparación con la experiencia cotidiana, los estudiantes tienden a utilizar el concepto de luz instantánea como un teorema en acción (Otero, Arlego y Prodanoff 2016).

Ante estos problemas, existe un consenso creciente en decantarse por un tratamiento geométrico y cuatridimensional de la TRR, no sólo en la cinemática, donde, como ya hemos dicho, conviene usar los diagramas espaciotiempo de Minkowski, sino también en la dinámica relativista, donde se pueden usar con el mismo propósito e igual rendimiento los diagramas impulso-energía (Taylor y Wheeler 1992). De este modo, hemos basado nuestra propuesta de enseñanza en una visualización geométrica adecuada de la teoría de la relatividad. Esto implica el reconocimiento de la masa como el módulo, en el sentido de Minkowski, del par vector momento-energía ( $\mathrm{p}, \mathrm{E})$. La misma formulación geométrica conduce a la visualización de la energía (y no solo de la masa), como la magnitud que define las propiedades inerciales de un cuerpo. La definición funciona tanto para una partícula como para un sistema de partículas. Otros autores han hecho propuestas del mismo tipo. Por ejemplo, Alonso y Soler (2006 b) elaboraron una secuencia didáctica para bachillerato, en la que se obtiene el vector dinámico (impulso-energía), a partir del cinemático (espacio-tiempo). Con este planteamiento las leyes y los conceptos básicos de la dinámica relativista se implementan sobre el entramado espaciotemporal y, como los de la cinemática, derivan de forma natural de los postulados de la TRR.

\section{Conclusiones}

A continuación, resumimos las principales conclusiones de los estudios revisados en el apartado anterior. De acuerdo con la línea que se indicó al final del mismo, las expondremos de forma que nos permitan establecer las implicaciones didácticas que nos han guiado en el diseño de la secuencia de actividades apropiada para la enseñanza y el aprendizaje de la TRR de Einstein usando la geometría de Minkowski.

\section{Espacio y tiempo}

Los estudiantes tienen dificultad para conceptualizar las características de continuidad del espacio y del tiempo que aparecen al tratar el concepto de velocidad límite:

En el caso de Galileo, la velocidad puede crecer indefinidamente. Esto significa que el límite es infinito o, simplemente, que no hay límite. 
En la TRR la velocidad también puede crecer constantemente, pero, en cualquier caso, no puede exceder de un valor determinado, el de la velocidad de la luz.

Los estudiantes también muestran una resistencia singular a aceptar que las medidas de longitud o de tiempo pueden depender del sistema de referencia, ya que atribuyen un carácter absoluto a las medidas realizadas en un sistema de referencia en reposo con respecto al observador. Se pone de manifiesto la persistencia del concepto de tiempo newtoniano, que fluye en todas partes por igual en todos los sistemas de referencia.

\section{Causalidad y Simultaneidad}

Existe un bajo compromiso epistemológico con las relaciones causa/efecto, así como ideas erróneas respecto a la simultaneidad. Nada se puede afirmar al respecto y se le atribuye un carácter absoluto o excesivamente relativo. Una confusión habitual es la que existe entre la simultaneidad de dos eventos y la recepción de las señales por parte del observador.

\section{Sistemas de referencia}

Los estudiantes otorgan un estatus diferente de realidad a las observaciones realizadas desde un sistema de referencia en reposo y otro en movimiento. Al reposo se le atribuye un carácter absoluto, y se produce confusión entre el observador y el sistema de referencia. Además, tienen dificultad para relacionar las gráficas de espaciotiempo con el movimiento que representan.

\section{Energía}

El alumnado considera que la energía es una entidad cuasi material y no existe conciencia de la influencia del sistema de referencia en la energía cinética y el trabajo mecánico. Tiene dificultades para admitir cambio de energía cuando cambia el sistema referencial.

En nuestra propuesta de enseñanza, se concede un papel primordial al sistema de referencia del centro de masas, por el hecho de que permite ver la simetría de las situaciones presentadas, lo que confirma la valía de este sistema de referencia particular.

\section{Masa}

Hay confusión entre masa y cantidad de materia, y se considera que la masa cambia con la velocidad (masa relativista). Este concepto suscitó una controversia en el ámbito científico, y actualmente se utiliza la noción de masa invariante, equivalente a la energía total en el sistema referencial en reposo con el centro de masas. El concepto se visualiza adecuadamente en el espaciotiempo de Minkowski. La definición operativa de masa inercial coincide con la definición anterior en los diagramas de espaciotiempo del choque inelástico.

\section{Luz}

Se cree que el carácter límite de la velocidad de la luz se debe a la insuficiencia de la técnica actual, mientras que la suma de velocidades de Galileo se mantiene. Existe confusión entre la invariancia de la velocidad de la luz y la característica de ser una velocidad límite. Además, se cree que las altas velocidades producen efectos destructivos sobre los objetos. Se atribuyen propiedades mecánicas a la velocidad de la luz en la fórmula de Einstein (que desaparecen cuando se usan unidades en las que $\mathrm{c}=1$ ). Las noticias en los medios sobre experimentos con velocidades supralumínicas en mecánica cuántica se interpretan erróneamente como refutaciones de la TRR.

Esta influencia de los medios de comunicación, que en ocasiones utilizan una terminología poco rigurosa, conduce a veces a confusión e ideas equivocadas. Sin embargo, la TRR es una 
de las teorías más precisas y completamente comprobadas y las tecnologías dependientes de ella tienen una enorme repercusión en la vida diaria.

\section{Implicaciones para la Enseñanza}

El hecho de que la mayoría de los libros de texto y enfoques disponibles para la enseñanza de la TRR se basen en su formulación matemática como recurso fundamental acrecienta las dificultades de los estudiantes para comprenderla, especialmente en las edades más tempranas (Kicilcik y Ünlü Yavas 2017).

El pensamiento de los estudiantes se enfrenta a menudo con las características contraintuitivas de la teoría de la relatividad. Con frecuencia, esto lleva a la opinión de que es un conocimiento solo al alcance de un reducido grupo de personas con elevada formación científica en física moderna. Esta opinión se consolida debido a:

El hecho de que, habitualmente, dicha teoría se presenta mediante formalismos matemáticos complejos: tensores, matrices, números complejos, cuadrivectores, etc.

Las ideas de los estudiantes —nociones de espacio y tiempo absolutos, energía nuclear asimilada a la combustión-, de tipo intuitivo, u otras que subyacen a comentarios más o menos habituales (todo es relativo) y hasta ideas seudocientíficas, muchas veces relacionadas con la ciencia ficción.

La gran dificultad que tienen los estudiantes para sustituir los conceptos de la mecánica newtoniana por los relativistas, por la falta de experiencias cotidianas concretas que les pudieran ayudar a dar credibilidad a la nueva teoría y comprobar su eficiencia.

De la discusión precedente se ha inferido que una propuesta de enseñanza basada en la característica esencialmente geométrica de la TRR, que permite presentar y resolver los problemas relativistas de forma visual, podría facilitar el aprendizaje de la misma. Basándose en la geometría, estas visualizaciones tienen las mismas características de precisión y generalidad que las formulaciones algebraicas.

Para ilustrar nuestra propuesta para la enseñanza de la TRR, presentamos el siguiente ejemplo de cómo deducir de forma visual las características geométricas de la transformación de Lorentz, que, como demostró Minkowski, constituye la base conceptual sobre la que se construye todo el edificio de la TRR.

Para comenzar, simplificamos el espaciotiempo real, de cuatro dimensiones (tres de espacio y una de tiempo) reduciéndolo a una dimensión espacial y otra temporal. Como es habitual en la TRR, situaremos el espacio en abscisas y el tiempo en ordenadas (figura 1). La distancia espacial OS determina la unidad de espacio $U_{e}$, y el intervalo de tiempo OB determina la unidad temporal $U_{\mathrm{t}}$. Estas unidades se establecen de forma que la velocidad de la luz (c) sea igual a la unidad: $c=U_{\mathrm{e}} / U_{\mathrm{t}}$ 
Cualquier par de valores de espacio y de tiempo que cumplan esta condición se llaman unidades naturales, y son imprescindibles para una correcta visualización geométrica de los fenómenos relativistas. Podemos así representar mediante un cuadrado (OALS) una celdilla unidad de espaciotiempo.

En la misma figura podemos reconocer las siguientes líneas:

-Línea OA: corresponde a un objeto en reposo (observador A) por el que transcurre una unidad de tiempo.

-Línea OB: corresponde al movimiento de un objeto (observador B) que se mueve hacia la izquierda con movimiento rectilíneo uniforme (mru).

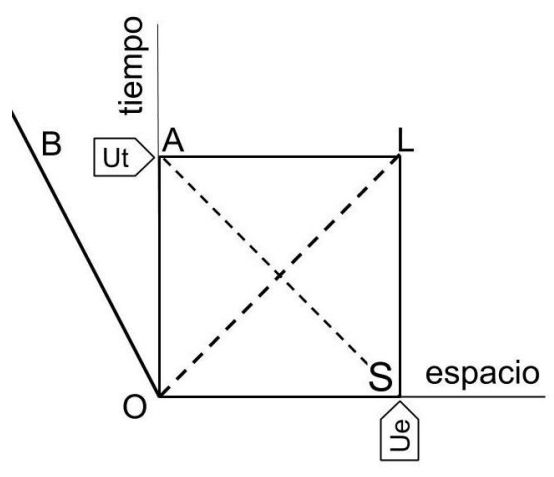

Figura 1. Celda unidad de espaciotiempo y líneas de movimiento. Observador (SRI) A

-Línea OL (de trazos): es la diagonal de la celdilla cuadrada unidad, corresponde a una partícula (un fotón) moviéndose a la velocidad de la luz $(c=1)$.

-Línea SA: es la otra diagonal y denota igualmente la velocidad de la luz, pero hacia la izquierda.

Vamos ahora a representar la misma situación, pero esta vez desde el punto de vista del móvil (observador) B. Para ello acudimos en primer lugar (y de forma muy sintética por razones de extensión) a una serie de consideraciones físicas que nos delimitan el tipo de transformación.

Las teorías relativistas consideradas contemplan transformaciones entre Sistemas de Referencia Inerciales (SRI). Geométricamente, esto se traduce en que toda línea recta (que, como vimos, se corresponde con un mru en el espaciotiempo) se transforma en otra línea recta.

Cualquier paradigma relativista implica también el principio de relatividad, por el cual los sucesos físicos se producen por igual para cualquier observador. En este caso en que los únicos movimientos son rectilíneos y uniformes, y por lo tanto se representan mediante rectas (figura 1), los sucesos físicos son las interacciones entre objetos (colisiones), y ello implica que las rectas paralelas se transforman en rectas paralelas (puesto que, si para un observador A dos objetos no colisionan, describen un par de rectas paralelas en el diagrama et, y por el principio de relatividad, tampoco colisionarán para otro observador $\mathrm{B}$, con lo que seguirán siendo paralelas para este).

Toda transformación de un plano que cumpla las anteriores condiciones será una transformación lineal, que transformará la celda unidad cuadrada en un paralelogramo, en cualquiera de los paradigmas relativistas.

El espacio físico es isótropo. Un observador en dicho espacio no puede diferenciar en qué dirección se mueve a partir de observaciones físicas. De esto se infiere que el área de la celdilla no puede cambiar en la transformación, pues si aumentase en un sentido disminuiría en el otro, y el espacio ya no sería isótropo.

En consecuencia, toda transformación relativista debe ser lineal y conservar las áreas del espaciotiempo.

Solo nos queda, ahora, aplicar lo dicho a nuestra situación inicial. 
Desde el nuevo punto de vista del observador B, el observador anterior (A) estará en movimiento, por lo que los laterales del cuadrado se transformarán en dos segmentos paralelos con una misma inclinación. Para completar el paralelogramo debemos establecer un postulado adicional referido a los lados horizontales del cuadrado inicial mostrado en la figura 1.

Esto fue resuelto por Galileo (figura 2) postulando un tiempo universal y absoluto para todos los observadores. Es decir, los lados horizontales del cuadrado seguirán siendo horizontales en el nuevo paralelogramo. La luz que iba hacia la derecha (de O a L) en la situación inicial (observador A) tiene ahora, para el observador B y en el paradigma galileano, una velocidad (tangente del ángulo BOL) mayor que c, mientras que la luz que va de $\mathrm{S}$ a $\mathrm{A}$ lo hace ahora a una velocidad menor que c. Como se ve, la velocidad de la luz para Galileo no es constante.

La física del siglo XX, a partir de Maxwell (de forma teórica) y Michelson (de forma experimental) ha demostrado que esto no se corresponde con la realidad, pues viola el carácter absoluto de la velocidad de la luz.

Para resolver este dilema, Einstein propuso incorporar el carácter absoluto de la velocidad de la luz como un postulado alternativo al del tiempo absoluto de Galileo, el cual, como vimos, implicaría una variación inaceptable en la velocidad de la luz. La luz debe tener ahora la misma velocidad para todos los observadores, es decir, las diagonales del cuadrado inicial OL y SA (figura 1) deben mantener la inclinación que tenían (por lo que seguirán siendo

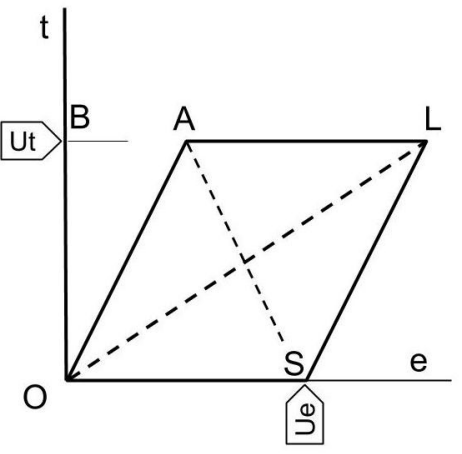

Figura 2. Transformación de Galileo. Observador B perpendiculares entre sî). El único paralelogramo cuyas diagonales son mutuamente perpendiculares es un rombo, y en nuestro caso sus diagonales deben tener una inclinación de $45^{\circ}$ respecto a la vertical (figura 3 ).

Además, como el área del rombo (que es la mitad del producto de sus diagonales) debe conservar su valor, al aumentar una de ellas (OL) debe disminuir la otra (SA) en igual proporción, llegando así a la forma definitiva de la transformación para la TRR, conocida como transformación de Lorentz.

Conviene no olvidar que, aunque de longitudes diferentes, los segmentos OL y SA tienen una misma inclinación (en valor absoluto) respecto de la vertical.

Tampoco debemos contemplar las figuras 1 y 3 como representaciones de distintos sucesos, sino que, como se dijo, corresponden ambas a un mismo suceso descrito por diferentes observadores.

Lo que tiene de particular la figura 3 frente a la figura 2 es que, ahora, esta transformación implica unas propiedades contraintuitivas pero realistas de las

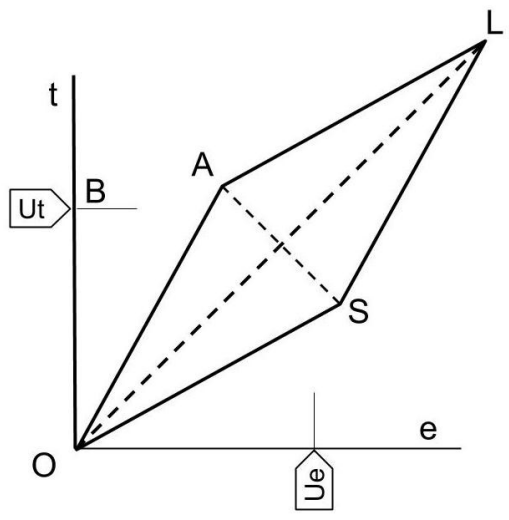

Figura 3. Transformación de Lorentz. Observador B magnitudes físicas implicadas. Comparando las figuras 1 y 3 podemos visualizar, por ejemplo, el fenómeno relativista de la dilatación temporal por la mayor altura (tiempo) que alcanzan las líneas OA y OL (mientras que en la figura 2 dichas alturas no variaban). 
Una comparación más meticulosa nos permitiría visualizar otros fenómenos relativistas como la contracción espacial o la velocidad límite.

Además, las características geométricas de la transformación realizada permiten resolver gráficamente situaciones de mayor demanda cognitiva como son, por ejemplo, la equivalencia entre masa y energía o el choque elástico (Prado, Area, Paredes, Domínguez-Castiñeiras, Edelstein y Mira 2018).

En el Bachillerato actual apenas se trabaja (más allá de mencionarlas) con las transformaciones de Lorentz, por lo que las consideraciones anteriores podrían ser aptas para los primeros cursos universitarios.

Sin embargo, aunque ahora mismo tanto el nivel, como el detalle en los desarrollos que hemos mostrado para derivar las transformaciones de Lorentz a partir de los diagramas espaciotemporales, excederían de lo exigido en el Bachillerato, sus características netamente visuales y su eficacia interpretativa a la hora de incorporar en los diagramas espaciotemporales las magnitudes físicas mencionadas, hacen plausible e interesante su toma en consideración en estas etapas previas. Por ello es preciso simplificar el rigor expositivo de desarrollos como el que hemos presentado anteriormente para adaptarlo a las capacidades cognitivas del alumnado, estableciendo así una secuencia didáctica adaptada al bachillerato (Prado Orbán 2012)

Nuestra propuesta, se diseñó, aplicó y evaluó en el aula con resultados prometedores para los niveles preuniversitarios (Prado Orbán y Domínguez-Castiñeiras 2010).

\section{Agradecimientos}

X. P. y J. M. D. C. hacen constar que el presente trabajo se enmarca en los proyectos ESPIGA (PGC2018096581-B-C22) y EAMARE-STEAM (EDU2017-84979-R) y agradecen la financiación al fondo Europeo de Desarrollo Regional (FEDER)/Agencia Estatal de Investigación del Ministerio de Ciencia, Innovación y Universidades. I. A. agradece la ayuda de los proyectos ED431B 2018/57, de la Consellería de Educación, Universidad y Formación Profesional (Xunta de Galicia) y FIS2017-83762-P, del Ministerio de Economía, Industria y Competitividad. Á. P. agradece la ayuda de los proyectos MTM2016-75140-P, de la Agencia Estatal de Investigación, co-financiado por fondos FEDER, y R 2016/022, de la Xunta de Galicia.

\section{Referencias}

Alemañ, R. A. (1997) Errores comunes sobre relatividad entre los profesores de Enseñanza Secundaria. Enseñanza de las Ciencias, 15(3), 301-307. URL

Alonso, M. (2000) Diagramas posición-tiempo para enseñar relatividad en el Bachillerato. Alambique. Didáctica de las Ciencias Experimentales. 22, 109-117. (Disponible en la Web de Materiales Didácticos de la Sección Local de Alicante) URL

Alonso, M. y Soler, V. (2002) Reflexions sobre la forma habitual d'introduir els conceptes de la dinàmica relativista en batxillerat. Actas de las Jornadas de enseñanza de las Ciencias de la AEFQ Curie. (Disponible en la Web de Materiales Didácticos de la Sección Local de Alicante) URL

Alonso, M. y Soler, V. (2006 a) La relatividad en el Bachillerato. Una propuesta de unidad didáctica. Enseñanz̧a de las Ciencias, 24(3), 439-454. URL

Alonso, M. y Soler, V. (2006 b) Materiales didácticos para enseñar y aprender relatividad en el Bachillerato. Alambique. Didáctica de las Ciencias Experimentales, 50, 106-115. URL

Aslanides, J.S. y Savage, C.M. (2013) Relativity concept inventory: Development, analysis, and results. Physical Review Physics Education Research, 9(1), 010118 (1-10). DOI 
Consello da Cultura Galega (2017) Verbo da teoría da relatividade restrinxida e xeral. Traducción de la obra original alemana Über die spezielle und die allgemeine Relativitätstheorie (Gemeinverständlich) de Albert Einstein (1916). Coordinador de la obra: Jorge Mira. Editorial: Consello da Cultura Galega. URL

Corry, L. (1997) Hermann Minkowski and the postulate of relativity. Archive for History of Exact Sciences, 51(4), 273-314. DOI

Díaz, A. D.; Herrera, W. J. y Manjarrés, D. A. (2009) Work and energy in inertial and noninertial reference frames. American Journal of Physics, 77(3), 270-273. DOI

Dib, C. (2013) Mass as a Form of Energy in a Simple Example. The Physics Teacher, 51(9), 546548. URL

Doménech, A. (1998) El debate sobre la masa relativista: el problema definicional y otros aspectos epistemológicos. Enseñanza de las Ciencias, 16(2), 331-339. URL

Doménech, A.; Doménech, T.; Cassasus, M. y Bella, T. (1985) El espacio tiempo clásicos y el espacio tiempo de la relatividad especial. Enseñanza de las Ciencias, vol. extra, parte 2, p. 72. URL

Doménech, A.; Casasús, E. y Doménech, M.T. (1993) The classical concept of mass: theoretical difficulties and students' definitions. International Journal of Science Education, 15(2), 163-173. DOI

Drummond, I. T. y Hathrell, S. J. (1980) QED vacuum polarization in a background gravitational field and its effects on the velocity of photons. Physics Review, D22 (2), 343-355. DOI

Fort, J. (2005) Energy conservation can predict intervals of time. Physics Education, 40(4), 321322. DOI

Galili, I. y Kaplan, D. (1997) Extending the application of the relativity principle: Some pedagogical advantages. American Journal of Physics, 65(4), 328-335. DOI

Gil, D. y Solbes, J. (1993) The introdution of modern physics: overcoming a deformed vision of science. International Journal of Science Education, 15(3), 255-260. DOI

Hartatiek, Y. y Dwi H. (2017) Effect of Tutorial Giving on the Topic of Special Theory of Relativity in Modern Physics Course Towards Students' Problem-Solving Ability. Journal of Physics: Conference Series, 846(1), 1-7. URL

Haug, E. G. (2017) The ultimate limits of the relativistic rocket equation. The Planck photon Rocket. Acta Astronautica 136, 144-147. URL

Hecht, E. (2009) Einstein Never Approved of Relatistic Mass. The Physics Teacher, 47, 336-341. DOI

Hecht, E. (2011) How Einstein confirmed $\mathrm{E}_{0}=\mathrm{mc}^{2}$. American Journal of Physics, 79, 591-600. DOI

Hewson, P. W. (1982) A case study of conceptual change in special relativity: The influence of prior knowledge in learning. European Journal of Research in Science Education, 4(1), 61-78. DOI

Hewson, P. W. (1990) La enseñanza de 'Fuerza y Movimiento' como cambio conceptual. Enseñanza de las Ciencias, 8(2), 157-171. URL

Hewson, P. W. y Thorley, N.R. (1989) The conditions of conceptual change in the classroom. International Journal of Science Education, 11 (special issue), 541-553. DOI 
Hobson, A. (2005) Teaching: mass without mass. The Physics Teacher, 43(2), 80-82. DOI

Hobson, A. (2006) Millikan Award Lecture, 2006: Physics For All. American Journal of Physics, 74(12), 1048-1054. DOI

Kicilcik, H. S. y Ünlü Yavas, P. (2017) Investigating the Reasons of Difficulty Understanding of Students in Special Relativity Topics. Cukurova Üniversitesi Eğitim Fakültesi Dergisi, 46(2), 399-426. DOI

Logunov A. A. (1998) Curso de Teoría de la Relatividad y de la gravitación. Moscú: Universidad Estatal de Lomonósov.

McDermott, L. C. (1993) How we teach and how students learn-A mismatch? American Journal of Physics, 61(4), 295-298. DOI

Minkowski, H (2012) Espacio y tiempo. Documentos de Minkowski sobre la relatividad (versión gratuita). Traducido por Fritz Lewertoff y Vesselin Petkov. Editado por Vesselin Petkov. Quebec, Montreal, Canadá: Minkowski Institute Press. URL

Naber, G. L. (2012) The geometry of Minkowski spacetime. An introduction to the mathematics of the special theory of relativity. 2nd ed. (English). Berlin: Springer. URL

Okun, L.B. (1989) The Concept of Mass. Physics Today, 42(6), 31-36. DOI

Otero, M.; Arlego, M. y Prodanoff, F. (2016) Teaching the basic concepts of the Special Relativity in the secondary school in the framework of the Theory of Conceptual Fields of Vergnaud. Il Nuovo Cimento C, 38(3), 1-13. DOI

Pérez, H. (2003) La teoría de la relatividad y su didáctica en el Bachillerato: análisis de dificultades y una propuesta de tratamiento. Tesis doctoral, Universitat de Valencia. URL

Pérez, H. y Solbes, J. (2003) Algunos problemas en la enseñanza de la relatividad. Enseñanza de las Ciencias, 21(1), 135-146. URL

Pérez, H. y Solbes, J. (2006) Implicaciones de la evolución histórica de algunos conceptos en la enseñanza de la relatividad. Revista Eureka sobre Enseñanza y Divulgación de las Ciencias, 3(3), 408-430. URL

Prado Orbán, X. y Domínguez-Castiñeiras, J.M. (2010) A Didactic Proposal for de Visual Teaching of the Theory of Relativity in High School First Course, pp. 297-305. En Taşar y G. Çakmakci (Eds.), Contemporary Science Education Research: Teaching. Pegem Akademi: Ankara, Turkey. ISBN: 9786053640301. URL

Prado Orbán, X. (2012) Deseño e implementación dunha proposta de ensinanza da relatividade especial en primeiro de bacharalato, baseada na formulación xeométrica de Minkowski. Universidade de Santiago de Compostela. Servicio de Publicacións e Intercambio Científico: Santiago de Compostela. URL

Prado, X.; Area, I.; Paredes, A.; Domínguez-Castiñeiras, J.M.; Edelstein, J.D. y Mira, J. (2018) Archimedes meets Einstein: a millennial geometric bridge. European Journal of Physics, 39(4), 045802. DOI

Riggs, P. (2016) A Comparison of Kinetic Energy and Momentum in Special Relativity and Classical Mechanics. The Physics Teacher, 54(2), 80-82. DOI

Salart, D.; Baas, A.; Branciard, C.; Gisin, N. y Zbinden, H. (2008) Testing the speed of 'spooky action at a distance'. Nature, 454(7206), 861- 864. DOI 
Scherr, E. R.; Shaffer, P. S. y Vokos, S. (2001) Student understanding of time in special relativity: Simultaneity and reference frames. American Journal of Physics, 69(7), 24-35. URL

Scherr, R. E.; Shaffer, P. S. y Vokos, S. (2002) The challenge of changing deeply held student beliefs about the relativity of simultaneity. American Journal of Physics, 70(12), 1238-1248. DOI

Serafin, K, y Glazek, S. (2017) Elementary example of energy and momentum of an extended physical system in special relativity. American Journal of Physics, 85(7), 529-541. URL

Tanel, Z. (2014) Student Difficulties in Solving Problems Concerning Special Relativity and Possible Reasons for these Difficulties. Journal of Baltic Science Education, 13(4), 573-582. URL

Taylor, J. L, (2001). Physics, Philosophy and the nature of space and time. Physics Education, 36(3), 207-211. DOI

Taylor, E. F. y Wheeler, J. A. (1992) Spacetime Physics. Introduction to Special Relativity. New York: W. H. Freeman and co.

Tefft, B. J. y Tefft, J. A. (2007) Galilean Relativity and the Work-Kinetic Energy Theorem. The Physics Teacher, 45(4), 218-220. DOI

Toledo, B.; Arriasseco, I. y Santos, G. (1997) Análisis de la transición de la física clásica a la relativista desde la perspectiva del cambio conceptual. Enseñanza de las Ciencias, 15(1), 79-80. URL

Tommasini, D.; Paredes, Á. y Michinel, H. (2019) Comment on "the ultimate limits of the relativistic rocket equation. The Planck photon rocket". Acta Astronautica, 16, 373-374. URL

Villani, A. y Pacca, J. (1987) Students' Spontaneous Ideas about the Speed of Light. International Journal of Science Education, 9(1), 55-65. DOI

Walter, S. (2008) Hermann Minkowski's approach to physics. Mathematische Semesterberichte, 55(2), 213-238. DOI

Warren, J.W. (1976) The mystery of mass-energy. Physics Education, 11(1), 52-54. DOI

Whitaker, M. A. B. (1976) Definitions of mass in special relativity. Physics Education, 11(1), 5557. DOI 